\title{
Preference effects on acquisition and retention of concurrent discriminations by rhesus monkeys
}

\author{
F. ROBERT TREICHLER, THOMAS V. PETROS, and SHARON A. LESNER \\ Kent State University, Kent, Ohio 44242
}

\begin{abstract}
Ten rhesus monkeys were trained on five tasks, each of which consisted of eight concurrently presented object discrimination problems. Sequences of presentation were devised to allow one, two, or three new tasks to intervene between acquisition and retention tests or to provide a 30-day period of no testing. Equivalent and proficient performances were obtained in all retention tests, and no relationship was observed between retention and the initial preference characteristics of various objects. Object preferences did produce significant influences upon acquisition, but these effects were not as pronounced in early tasks as in later ones. An additional retention test provided support for the contention that monkeys do not necessarily process information about specific object pair discriminations. Rather, they appeared to retain a list of previously rewarded objects even when object pairings were different from those provided during acquisition. Concurrent discriminations involving many distinct objects were resistant to interference and independent of preference characteristics over long retention periods.
\end{abstract}

Bessemer and Stollnitz (1971) have provided an interpretation of the monkey's retention of two-choice discriminations that emphasizes the role of preferences shown on the first exposure to the objects that serve as discriminanda. It was their contention that an animal's memory for particular problems could be predicted from the choice behavior on an initial onetrial presentation of those discriminanda. If that choice were rewarded (this was ensured by placing food under both objects), retention was superior to that seen if the initial choice were nonrewarded (no food was placed under either object, but in subsequent trials, food was under the initially unchosen one). The conditions were designated as baited and unbaited, and the manipulation was considered to provide problems for which solution required selection of preferred objects in the former case or nonpreferred objects in the latter. Furthermore, such preferences were said to influence retention under both consecutive and concurrent procedures of problem presentation.

Treichler, Wetsel, and Lesner (1977) conducted several retention tests with the concurrent discrimination procedure and observed that preference manipulation via differential first-trial reward yielded significant influences when ease of acquisition on eight concurrent problems was measured. However, retention of those problems after one intervening eightproblem task was not related to these preference characteristics. Indeed, retention performance was uniformly good (modal errors in retention $=0$ ) for problems that had been either rewarded or nonrewarded on their first presentation. These authors speculated that object preferences might affect acquisition, but when once learned, the problems were no longer subject to preference influences.
However, it could be argued that the interference from only one intervening eight-problem task did not produce enough error to allow for the expression of differential preference effects. One aim of the present study was to provide varied amounts of information between acquisition and a retention test in an effort to obtain sufficient error for measuring retention differences when problems required choice of preferred or nonpreferred objects.

The need for large numbers of problems to serve as interfering information between acquisition and retention in the present study allowed a test of another issue concerning the monkey's memory for discriminations. Medin (1977) reviewed evidence on the relationship between retention and the extent of prior discrimination training. Although there was little generality of results, he did note instances in which some retention measures were better after learning a relatively small number of problems than they were after the animals had undergone extensive training. Because the present investigation required animals to learn 40 different problems, an opportunity was afforded to compare both the acquisition and the retention of problems learned early or late in the series. Furthermore, in keeping with our concern for the effects of preference characteristics upon discrimination, it was possible to measure whether or not any performances were systematically related to the requirement of choosing preferred or nonpreferred objects for problem solution.

The purpose of the present study was to assess the role of preferences, as expressed by choices made on first exposure to objects, upon acquisition and retention of concurrent discrimination tasks. Each of these tasks comprised eight problems, and the tasks 
appeared in sequences that allowed evaluation of both their serial order of presentation and the effects of varied amounts of interference between acquisition and retention testing.

\section{PHASE 1}

\section{Method}

Ten naive adolescent rhesus macaques ( 2.6 to $4.2 \mathrm{~kg}$ ) served as subjects. All were adapted to the WGTA and were trained to displace common-use multidimensional objects of the kind used by Treichler et al. (1977). Soybeans, sugar pellets, or raisins served as rewards. Initial pretraining consisted of the presentation of one object pair until a criterion of no error on 10 consecutive noncorrectional trials was achieved, and 10 such problems were administered. The concurrent discrimination procedure was then introduced in a further pretraining procedure that provided four different problems with trial sequences mixed in varied orders. Eight trials (twice through the list of four problems) were presented each day, and testing was continued until a subject was correct on all trials in a daily session.

In the experiment proper, all subjects were assigned in an unbiased fashion to one of two groups. Both groups received a series of acquisition and subsequent retention tests on five different concurrent eight-problem tasks. Table 1 displays the order of the acquisition and retention testing sequence for each group. The consecutive letters indicate the order of presentation of tasks. These orders were designed to allow each group to have an acquisition and retention test separated by one, two, and three intervening new tasks. The use of specific sets of eight object pairs as tasks was systematically counterbalanced over the sequence for the five subjects within each group in order to avoid confounding of task and order effects.

The daily testing session for a subject entailed presentation of 16 trials. In these sessions, each of the eight concurrent problems appeared in one order and then in a different order. Testing was continued for each subject until a criterion of no intrasession error was achieved or until 15 days of training had been completed. Failure to achieve the errorless intrasession criterion was very rare (in only 2 of 50 acquisitions and once in retention). Each subject proceeded through the tasks in their prescribed order, and each new task was begun on the day following criterion meeting on the previous task. After completion of all acquisition tests and the first three retention tests, a 30-day period of no testing was imposed. Retention tests on the remaining two tasks were presented at the end of this period. In only a few instances did the failure of environmental control equipment and/or the

Table 1

The Series of Acquisition and Retention Tests Given to Two Groups of Five Subjects

Group 1 30 Days $\quad$ No Test

Note-Letters indicate the various eight-problem tasks, and the subscript (2) indicates retention tests. The task sequence proceeds from left to right, and numbers associated with brackets indicate the number of intervening new tasks between acquisition and retention. illness of trainers prevent adherence to a schedule of daily test administration.

Whenever acquisition of a new task was undertaken, the first trial of four randomly selected problems was shown to the animal with food in both food wells, while, on the other four problems, no food was placed in either well. This procedure allowed for the subsequent analysis of the conditions that have been presumed to reflect preference characteristics and have been designated "baited" (Bt) and "unbaited" (UBt) by Bessemer and Stollnitz (1971).

\section{Results}

The primary dependent variable in all analyses was the number of errors required to attain a criterion of no error within a 16-trial daily session.

Acquisition. One preliminary analysis of acquisition results was conducted to assess some possible confounding factors arising from our test procedures. The influences of assignment to the two different test groups (see Table 1) and the effects of the use of the five different groups of 16 objects (although counterbalanced) were compared for both baited and unbaited problems. In this 2 by 5 by 2 factorial, only the main effect of baited (Bt) vs. unbaited (UBt) problems was significant $[\mathrm{F}(1,8)=11.49, \mathrm{p}<.01]$, and neither the different test sequences nor the use of different object problems yielded any main effects or interactions (all ps $>.1$ ). The overall indication was that group assignment could be disregarded as a factor and that the sets of specific objects did not generate confounding effects in the design.

The principal evaluation of acquisition performance was done via a 2 by 5 analysis of variance involving a comparison of the consequences of serial order of tasks (A first, B second, C third, etc.) for both the Bt and UBt problems within these tasks. While no main effect of the serial order of tasks was evident $[F(4,36)=2.02, p>.05]$, a significant difference between the $\mathrm{Bt}$ and UBt problems was revealed $[F(1,36)=12.11, p<.01]$. Additionally, a marginal interaction of these two factors was indicated $[F$ $(4,36)=2.44, p=.065$ ] . The subsequent application of Newman-Keuls comparisons revealed that although no differences between $B t$ and UBt problems were evident in the first three tasks (A, B, C), significant differences (ps $<.01$ ) between $\mathrm{Bt}$ and UBt problems did exist in the last two acquisitions ( $D$ and $E$ ). As indicated in Table $2 a$, whenever significant differences between problem types were obtained, greater error was associated with UBt problems. The pattern of errors disclosed over the course of the five acquisition tasks suggested that the $\mathrm{Bt} / \mathrm{UBt}$ differences that have been so characteristic of acquisition by sophisticated monkeys (Bessemer \& Stollnitz, 1971; Treichler et al., 1977) might not be similarly displayed in the performances of naive animals.

Retention after intervening tasks. Table $2 b$ displays error scores for relearning to criterion with conditions of one, two, or three intervening new tasks. 
Table 2

Mean Errors-to-Criterion Scores in Acquisition for Baited and Unbaited Problems Through the Five-Task Sequence (a) and in Retention for Conditions Involving One, Two, or Three Intervening New Tasks (b)

\begin{tabular}{lccccccccc}
\hline & \multicolumn{4}{c}{} & \multicolumn{2}{c}{ (a) Task } & & & \multicolumn{3}{c}{ (b) Intervening New Tasks } \\
\cline { 2 - 9 } & $\mathrm{A}$ & $\mathrm{B}$ & $\mathrm{C}$ & $\mathrm{D}$ & $\mathrm{E}$ & & 1 & 2 \\
\hline Baited & 15.5 & 19.2 & 9.4 & 14.9 & 12.0 & 5.1 & 5.7 & 2.1 & 3 \\
Unbaited & 20.4 & 23.6 & 12.3 & 28.3 & 22.2 & 4.9 & 4.2 & 3.9 \\
\hline
\end{tabular}

A 3 by 2 analysis of variance indicated no significant effect of the number of intervening tasks for either Bt or UBt problems (all ps $>.05$ ). Additionally, a series of acquisition-retention comparisons for $\mathrm{B} t$ and UBt problems at each intervening task condition was conducted. In each case significant differences between retention and acquisition error were indicated (there were savings). Furthermore, although there were no main effects of $\mathrm{Bt} v \mathrm{vs}$. UBt problems, in two of the three tests, a significant interaction of $\mathrm{Bt} / \mathrm{UBt}$ and acqusition/retention measures was observed. Subsequent Newman-Keuls comparisons revealed that these results were due to greater acquisition error for UBt than Bt problems, while such differences did not exist in retention (all ps $<.05$ ).

Some other aspects of the strikingly good retention performance should also be noted. The imposition of intervening new problems and retention tests resulted in rather long time periods between acquisition and retention tests (mean days for one intervening = 9.8, for two intervening $=17.2$, and for three intervening $=27.4$ ), but retention performances showed very low levels of error and a lack of relationship to intervening training. For example, no retention error was observed on $41.3 \%$ of the problems after one intervening task, on $35.0 \%$ of the problems after two tasks, and on $42.5 \%$ of the problems after three tasks. If a retention criterion allowing just one error was set, then $76.3 \%, 56.0 \%$, and $67.5 \%$ of the retention test problems were solved under the respective intervention conditions noted above. Similarly, if proportion correct on the first trial of the retention tests was compared, all three intervention conditions yielded values of approximately .70 .

Retention after a period of no testing. Two further retention tests were conducted after a 30 -day period of maintenance in the home cages with no testing. This period began after completion of the last retention test of the intervening task conditions. Thirty days later, each animal received a relearning retention test on Task E (its last acquisition), and, after achieving criterion on this task, subjects were tested on the one other task (C or D) that had not been presented previously.

Table 3 displays median errors to criterion and numbers of subjects in various error categories for all the acquisition and retention tasks in the present study. The format of Table 3 also allows some comparison of the variability of scores obtained on each task. Section $3 b$ gives information for the two retentions conducted after the pause in testing. It may be noted that, although there are slightly higher median error scores and a few more subjects in the higher error categories than there were in the three initial retention tests, there were no significant differences among the five retention tests, according to a Friedman

Table 3

Median Acquisition and Retention Scores and Numbers of Subjects (Total $=10$ ) Assignable to Categorical Divisions of Errors to Criterion for the Various Tasks

\begin{tabular}{|c|c|c|c|c|c|c|c|c|}
\hline \multirow[b]{4}{*}{ Task } & & & \multicolumn{6}{|c|}{ Number of Subjects in Indicated Category } \\
\hline & & & \multicolumn{6}{|c|}{ Number of Errors to Criterion } \\
\hline & \multicolumn{2}{|c|}{ Median Errors to Criterion } & \multicolumn{2}{|c|}{$0-15$} & \multicolumn{2}{|c|}{$16-30$} & \multicolumn{2}{|c|}{31 or More } \\
\hline & Acquisition & Retention & Acquisition & Retention & Acquisition & Retention & Acquisition & Retention \\
\hline & \multicolumn{8}{|c|}{ (a) One, Two, or Three Intervening Tasks } \\
\hline 1 & 35.5 & 8.0 & 2 & 8 & 2 & 1 & 6 & 1 \\
\hline 2 & 23.5 & 7.0 & 2 & 6 & 5 & 3 & 3 & $i$ \\
\hline \multirow[t]{2}{*}{3} & 39.5 & 5.5 & 1 & 9 & 0 & 1 & 9 & 0 \\
\hline & \multicolumn{8}{|c|}{ (b) 30 Days of No Testing } \\
\hline $\mathrm{E}$ & 33.5 & 10.5 & 2 & 6 & 3 & 2 & 5 & 2 \\
\hline \multirow[t]{2}{*}{$\mathrm{D}$ or $\mathrm{C}$} & 29.5 & 14.5 & 3 & 6 & 2 & 4 & 5 & 0 \\
\hline & \multicolumn{8}{|c|}{ (c) Phase 2} \\
\hline AB Shuffled & 35.0 & 13.5 & 1 & 5 & 1 & 5 & 8 & 0 \\
\hline AB Maintained & 41.0 & 15.0 & 0 & 5 & 2 & 2 & 8 & 3 \\
\hline
\end{tabular}

Note-These include tesis (a) after different numbers of intervening tasks, (b) after 30 days of no testing, and (c) Phase 2 , a second retention of the first two acquisitions. 
analysis $\left[\chi_{\mathrm{r}}^{2}(4)=3.3, p>.05\right]$. Inspection of the raw data showed that, as in the three initial tasks, there was no differential retention of problems that were initially presented by the Bt or UBt procedures. The slightly greater retention error shown in these two tasks is attributable to the scores of only two or three animals, and this characteristic served as the rationale for summarizing via medians rather than means as was done in the earlier tests. Although a few subjects were slow to resume choice responding after the time down, other animals did begin immediately and demonstrated excellent retention. Four instances of scores of 0 or 1 error per task were obtained in these tests.

\section{PHASE 2}

Because proficient retention was observed on all five of the initial tasks and because no systematic relationship to preference characteristics was revealed in these tests, it was speculated that an even more difficult memory test might be required to provide a discriminative evaluation of retention. Accordingly, in Phase 2, animals were tested on retention of some variations of the first two tasks they had learned. This represented an attempt to provide both difficult retention tests and a comparison of procedures that have been associated with different information processing characteristics in concurrent discrimination. These procedures were originally used by Leary (1957) and were termed in the "pairs-shuffled" and "pairs-maintained" techniques. One contention has been that subjects using the pairs-shuffled procedure learn the several problems simply by retaining a list of correct objects, while subjects using the pairsmaintained procedure may rely on the contrast of stimulus properties within specific object pairs. Treichler et al. (1977) reported no differential effect of these procedures upon retention, although, in confirmation of Leary (1957), slight but significant influences of the manipulation were found in acquisition. However, in both previous studies, maintained vs. shuffled comparisons were based on differential treatments during acquisition or during acquisition and retention. In the present situation, all acquisition was conducted under the pairs-maintained procedure, and then, during retention, the method of presentation was either kept the same or changed to the pairsshuffled technique. A part of the rationale for this comparison was an interest in determining whether or not changes in presentation methods might represent a manipulation of "context" as a factor in memory (see Spear, 1978, chap. 2).

\section{Method}

The same subjects as those used in Phase 1 were tested. The 16 problems that had been presented in both Tasks $A$ and $B$ (see Table 1) were used to generate two further retention tasks.
Four problems from the A task and four from the B task (two Bt and two UBt from each task) were combined to comprise one retention test. These were presented with the same specific pairing of stimulus objects (correct and incorrect objects in combination) as had appeared in both the acquisition phase and the initial retention test. This set of eight object pairs comprised a test condition labeled "pairs-maintained." The original objects of the remaining eight object pairs (four from Task $A$ and four from Task B) were recombined so that correct objects now appeared with incorrect objects different from those with which they had been paired previously. This task was designated as a "pairsshuffled" retention. On retention test trials after the first one, still different combinations of correct objects with any of the incorrect ones might occur.

Testing was conducted as in Phase 1 , and the pairs-maintained and pairs-shuffled tests were given to different animals in counterbalanced order. Phase 2 testing began immediately after completion of those retentions that followed the pause in testing. This provided an average of 64 days between Phase 1 and Phase 2 retention tests on equivalent problems, and at least three other tasks were presented during this interval.

\section{Results}

Table $3 \mathrm{c}$ shows median acquisition and retention errors-to-criterion scores and the distribution of error scores for Phase 2 tests under both presentation procedures. A Wilcoxon comparison indicated no significant differences between retention scores $(T=$ $11.5, \mathrm{p}>.05)$ under the maintained and shuffled procedures. Additionally, a Friedman analysis of the two Phase 2 retentions and the three original retentions (one, two, or three intervening) yielded no significant differences $\left[\chi_{r}^{2}(9)=6.47 . p>.10\right]$ among these scores. In a comparison of retention and acquisition scores using Wilcoxon tests, significant savings were indicated when both the maintained $(T=7, p<.05)$ and the shuffled $(T=0, p<.01)$ procedures were used. Inspection of the data showed that retention error scores were unrelated to whether or not initial problems had been presented with the Bt of UBt procedures.

Phase 2 outcomes indicated that retentions were neither less proficient nor more highly related to baiting conditions than those seen in Phase 1. Furthermore, retention was not dependent upon maintaining the object pairs used during original task presentation, and no evidence for context influences was offered in the present situation. Independence from pair influences also lends support to the contention of Treichler et al. (1977) that concurrent task solutions characteristically entail learning a list of correct objects. Phase 2 retentions suggests that monkeys do not learn object pairs; rather, they retain information about correct objects and use this as a basis for their choices even when different incorrect objects appear in later pair combinations.

\section{DISCUSSION}

Several properties concerning the retention of concurrently learned discriminations were demonstrated in the present series of tests. 
First, good retention occurred under a variety of different conditions that were imposed in the interval between acquisition and subsequent memory test. Little difference was noted when varied numbers of new problems intervened or when a 30-day period of no testing was presented. Modal performances suggested that memory for concurrent discriminations is relatively independent of retroaction influences when many, distinctive, multidimensional objects serve as discriminanda.

Second, the role of initial object preferences in acquisition and retention of concurrent problems, which has been considered by Bessemer and Stollnitz (1971) and by Treichler et al. (1977), was further elucidated. When preference characteristics were manipulated by the baiting procedures present on the first trial, there were significantly fewer errors in the overall acquisition of $\mathrm{Bt}$ than of UBt problems. However, this pattern was not manifest in any tests of retention.

The present investigation's aim of producing a sufficient amount of error to evaluate differential retention of problems requiring choice of preferred or nonpreferred objects was not achieved, but the use of relearning tests did reveal some properties of the preference/retention relationship. There were some subjects that showed high error scores in a few retentions. In their performances, greater UBt than Bt error was seen, and the magnitude of their error scores was about the same as that of original learning. Thus, it appeared that these tests were the equivalent of presenting a new task, and the generalization that preferences influence acquisition, but not retention, seemed to be supported.

One other aspect of preference influences upon acquisition performance merits concern. Recall that a marginal interaction of serial task order and the $\mathrm{Bt}$ vs. UBt initial-trial condition was obtained in Phase 1 . Subsequent patterns of significance in a posteriori tests revealed that baiting conditions did not produce the typical relative ease of $\mathrm{Bt}$ problems on acquisition in the early tasks; however, this property did appear on the later tasks. Such an outcome suggests that monkeys may not come to simultaneous twochoice discrimination situations with compelling propensities to choose or avoid one of the discriminanda. Rather, differential selection characteristics develop with experience on the task. While the influence of object preferences upon acquisition is certainly a reliable feature of discrimination performance, the present finding suggests that it is probably inappropriate to regard such preference effects as simple "error factors" that are static, predetermined response tendencies to the physical features of particular objects. Although the present investigation was not designed to make formal comparison of performance differences in naive and sophisticated animals, it is suggested that future research efforts might consider changes in preference influences over a course of discrimination training.

In overview, the results from our series of tests are in accord with reviews (Bessemer \& Stollnitz, 1971; Medin \& Davis, 1974) that have characterized monkey memory as proficient. In the concurrent situation, this retention proficiency does not seem sternly related to the choice behaviors that a monkey has displayed on its first exposure to discriminanda. Furthermore, proficiency is not readily reduced by providing retroaction in the form of other distinctive object problems. However, results from concurrent transfer tests conducted by Sledjeski and French (1968) suggest that retention proficiency can provide a basis for rather substantial proactive interference effects when reward contingencies are changed between acquisition and transfer testing. In their experiments, as in the present Phase 2 results, the primary feature that seemed to characterize learning a number of concurrently presented problems was identification of a set of correct objects. Continued concern for the information processing characteristics used in retention of concurrent discrimination seems warranted, because this task provides a method for evaluating long-term memory processes that may parallel those underlying human multiple-problem retention.

\section{REFERENCES}

Bessemer, D. W., \& Stollnitz, F. Retention of discriminations and an analysis of learning set. In A. M. Schrier \& F. Stollnitz (Eds.), Behavior of nonhuman primates (Vol. 4). New York: Academic Press, 1971.

LEARY, R. W. The effect of shuffled pairs on the learning of serial discrimination problems by monkeys. Journal of Comparative and Physiological Psychology, 1957, 50, 581-584.

MEdiN, D. L. Information processing and discrimination learning set. In A. M. Schrier (Ed.), Behavioral primatology: Advances in research and theory (Vol. 1). Hillsdale, N.J: Erlbaum, 1977.

Medin, D. L., \& Davis, R. T. Memory. In A. M. Schrier \& F. Stollnitz (Eds.), Behavior of nonhuman primates (Vol. 5). New York: Academic Press, 1974.

Sledjeski, M., \& French, G. M. Multiple transfer paradigms following discrimination problem solution in monkeys. Journal of Comparative and Physiological Psychology, 1968, 66, 228-231.

SPEAR, N. E. The processing of memories: Forgetting and retention. Hillsdale, N.J: Erlbaum, 1978.

Treichler, F. R., Wetsel, W. C., \& Lesner, S. A. Some characteristics of concurrent discrimination and retention by monkeys. Learning and Motivation, 1977, 8, 213-228.

(Received for publication November 6, 1980; revision accepted February 18, 1981.) 